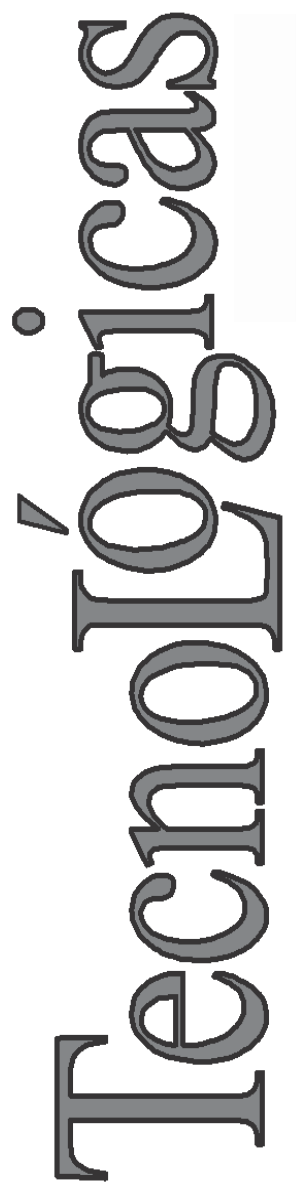

\title{
Sistema De Shearografía para el Análisis Dinámico de Materiales bajo Estrés Térmico
}

\section{Shearographic System for Dynamic Analysis of Materials under Heat Stress}

\author{
Nelson A. Correa-Rojas ${ }^{1}$ \\ Jesús D. Causado-Buelvas ${ }^{2}$ \\ Jorge A. Herrera-Cuartas ${ }^{3}$
}

1 Departamento de Electrónica y Telecomunicaciones, Instituto Tecnológico Metropolitano, Medellín-Colombia nelsoncorrea@itm.edu.co

2 Escuela de Física, Universidad Nacional de Colombia sede Medellín, Medellín-Colombia causado@gmail.com

3 Departamento de Electrónica y Telecomunicaciones, Instituto Tecnológico Metropolitano, Medellín-Colombia jorgeherrera@itm.edu.co 


\section{Resumen}

Los sistemas de shearografía son herramientas para el control e inspección de fallas e imperfecciones estructurales en diferentes tipos de aplicaciones industriales, automotrices y aeronáuticas. Se basan en la correlación digital de dos frentes de onda de patrones de speckle en dos estados de interés: con y sin carga. La técnica tiene la cualidad especial de ser autoreferenciada en sus frentes de onda y por tanto es muy robusta frente a perturbaciones ambientales. Se presenta un sistema shearografía en el cual se puede analizar el comportamiento dinámico de los esfuerzos que sufre un material en función de cambios en temperatura a lo largo de todo el proceso de carga térmica.

\section{Palabras clave}

Shearografía; ensayo no destructivo; metrología óptica; transformada de Hilbert

\section{Abstract}

Shearography is a tool for monitoring and inspecting of structural flaws and imperfections in different types of industrial, automotive and aeronautics applications. It is based on digital correlation of two speckle patterns in two states of interest: with and without load. The technique has the special quality of being very robust against environmental disturbances. We present a shearographic system to analyze the dynamic behavior of the strain that suffers a material in response to changes in temperature throughout the thermal load process.

\section{Keywords}

Shearography; nondestructive testing; optical metrology, Hilbert transform. 


\section{INTRODUCCIÓN}

El término shearografía es la españolización del inglés shearography una técnica láser de interferometría de patrones de speckle que es utilizada en la medición de deformaciones y esfuerzos en objetos que están sometidos a alguna clase de tensión. Esta técnica se caracteriza por realizar medidas de campo completo, sin contacto y de forma no destructiva, sobre un esquema de interferencia autorreferenciada.

La shearografía utiliza la superposición de un patrón de speckle con sí mismo, desplazado lateralmente una pequeña cantidad, de tal forma que no es necesaria la utilización de haces referencia para lograr un interferograma, como es en el caso de los sistemas holográficos. De esta forma, se tiene un sistema capaz de operar en condiciones de ambientes industriales, siendo inmune a notables cantidades de vibración y contaminación lumínica, que eran las principales limitantes de otras técnicas de metrología óptica de base holográfica. Igualmente, la shearografía no es exigente en las cualidades de coherencia del haz de iluminación, lo que permite su implementación utilizando láseres de semiconductor.

Las cualidades únicas de la shearografía, su montaje simple, su habilidad para operar en medios industriales y la capacidad de medir directamente la deformación de áreas de interés, la han convertido en una herramienta muy empleada en la industria aeroespacial (Krupka et al., 2005; Neuman, 2008) automotriz (Osten, 2003), entre otras (Kalms, 2006; Lobanov et al., 2007; Scalea et al., 2000), para el análisis de deformación, esfuerzos y la detección de fallas y defectos en estructuras, principalmente de materiales compuestos (Hung \& Ho, 2005; Hung, 1996).

La técnica se basa en la comparación de dos estados del objeto a estudiar: un estado inicial de referencia y un estado final, en el cual se encuentra sometido el cuerpo a alguna clase de tensión. Esta tensión provoca deformaciones que ponen en evidencia posibles defectos de la estructura como fracturas, delaminaciones, golpes, fatigas, entre otros. Tanto el estudio de las deformaciones y esfuerzos, como el monitoreo de defectos y fallas, son de gran inte- 
rés y utilidad en muchos campos de la ingeniería, y pueden ser realizados cualitativa y cuantitativamente.

Los sistemas convencionales solo realizan medidas estáticas, entre dos estados fijos estacionarios del objeto de estudio. Aunque estos sistemas son útiles, es más interesante realizar medidas de los procesos enteros de deformación, incluyendo los estados intermedios, que permiten apreciar dinámicamente los esfuerzos, los daños y defectos. Este trabajo presenta la implementación de un sistema de shearografía para el análisis dinámico cualitativo de deformaciones producidas por carga térmica. Se utilizan herramientas de análisis temporal que simplifican notablemente los cálculos convencionales que se realizan en el dominio espacial. Se obtiene la evolución de la fase a través del tiempo, la cual es proporcional a la deformación sufrida y al esfuerzo. Este sistema permite la detección de zonas de deformación crítica, el diseño de refuerzos y el control de defectos y fallas que solo sean notables a ciertos rangos de tensión.

En primer lugar se describen los principios generales de la técnica shearográfica para la medición de esfuerzos. Luego se presentan los métodos para el análisis dinámico de deformaciones a través del análisis temporal y la extracción de la fase utilizando las propiedades de la transformada de Hilbert. Finalmente, se muestra la metodología utilizada y los resultados obtenidos y su análisis.

\subsection{Principios Generales de Shearografía}

Un sistema de shearografía está constituido por un haz láser expandido que ilumina uniformemente la región de interés; un módulo óptico que recoge el campo reflejado por el objeto y lo superpone con él mismo, desplazado lateralmente una pequeña cantidad, para formar un patrón de interferencia de speckle que se registra con una cámara CCD y luego es procesado.

En la Fig. 1 se muestra la configuración más utilizada para shearografía. Un haz láser se expande con una lente para iluminar el área de interés uniformemente. Las microrrugosidades de la superficie provocan un reflejo granulado, llamado convencionalmente patrón de speckle. Este patrón es llevado a un interferóme- 
tro Michelson en donde es dividido en dos por un divisor de haz. Uno de los patrones es desplazado lateralmente una distancia $\delta x$ por medio de una ligera inclinación en uno de los espejos del interferómetro (espejo E2), y luego se combinan para interferir en el sensor de la cámara. También se muestra que, para realizar la medición, el objeto a estudiar se hace pasar de un estado inicial de referencia a uno final, por medio de la aplicación de carga mecánica o térmica, la cual provoca la deformación y la aparición de defectos o fallas.

La cámara CCD utiliza un sistema formador de imágenes para registrar el patrón de interferencia. Se hace una captura del estado referencia, se aplica la carga al objeto, y luego se hace un nuevo registro del estado final. La comparación de los dos estados se hace simplemente a través de la resta digital de las dos imágenes. El resultado de la resta es una imagen que contiene un conjunto de franjas, las cuales poseen la información sobre la deformación sufrida por el cuerpo.

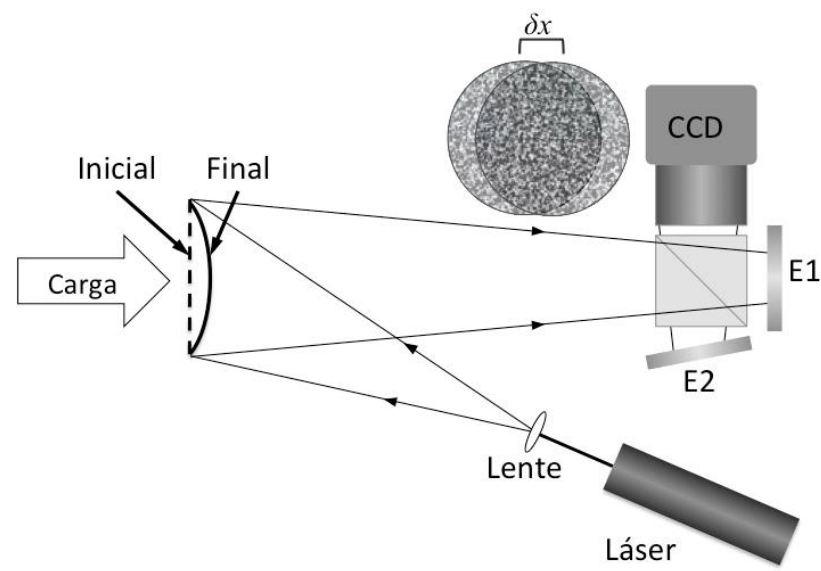

Fig. 1. Esquema de un sistema de shearografía. Un interferómetro Michelson recoge el patrón de speckle reflejado por el objeto y lo hace interferir con él mismo desplazado lateralmente por la acción de una pequeña inclinación en el espejo E2

Las franjas obtenidas pueden mostrar cualitativamente información respecto a la deformación o a defectos. Sin embargo, es la fase portada por las franjas la que realmente es importante, pues- 
to que es proporcional a las componentes de deformación, en el plano y fuera de él, y de esta forma, a los esfuerzos. Los factores de proporcionalidad están definidos por la longitud de onda del láser, la geometría de la configuración del sistema y las propiedades mecánicas del material (Steinchen \& Yang, 2003). La dirección en la cual se realiza el desplazamiento entre los dos patrones de speckle que interfieren, es decir $\delta x$, define la dirección de la derivada del desplazamiento del objeto, respecto a la cual se miden las deformaciones.

Los sistemas comunes de shearografía solo analizan un estado final deformado respecto al estado referencia. En éstos, es posible extraer apropiadamente la fase, sin embargo, se requiere que ambos estados sean lo suficientemente estables como para poder aplicar técnicas como el corrimiento de fase y el uso de fases portadoras determinadas. Por otra parte, existen muchas aplicaciones en las cuales es importante el análisis de los estados intermedios. Es decir, de todo el proceso de deformación, con lo cual el potencial de la técnica es mucho mayor.

\subsection{Fundamentos de Shearografía Dinámica}

Los principio teóricos de la shearografía y cómo la fase de un shearograma es proporcional a la deformación, es decir a la derivada del desplazamiento, está muy bien desarrollada y establecida (Steinchen \& Yang, 2003). El problema a resolver es la determinación instantánea de la fase en una secuencia de patrones de speckle que dan razón de la deformación de un cuerpo en un proceso dinámico de carga térmica. Para esto, tomamos la secuencia de patrones capturados durante el proceso. Cada uno de los cuadros de esta secuencia es restado con el cuadro inicial o referencia para obtener una nueva secuencia con patrones franjeados como los que se muestran en la parte derecha de la ¡Error! No se encuentra el origen de la referencia.. En este punto, la intensidad de cada uno de los patrones franjeados en cada cuadro de la secuencia se pueden describir matemáticamente de la siguiente forma (Madjarova et al., 2003):

$I\left(x, y, t_{i}\right)=I_{0}\left(x, y, t_{i}\right)+I_{m}\left(x, y, t_{i}\right) \cos \left(\phi\left(x, y, t_{i}\right)\right), i=1,2,3, \ldots$ 
Donde $I_{0}\left(x, y, t_{i}\right)$ e $I_{m}\left(x, y, t_{i}\right)$ son las intensidades de fondo y de modulación, respectivamente. Además, $\phi\left(x, y, t_{i}\right)=\theta\left(x, y, t_{i}\right)+$ $\varphi\left(x, y, t_{i}\right)$ es la fase de la señal, en la cual $\theta\left(x, y, t_{i}\right)$ es una fase aleatoria propia del speckle, y $\varphi\left(x, y, t_{i}\right)$ es la fase producida por la deformación del objeto, la cual varía lentamente en los dominios espacial temporal. $t_{i}$ es el tiempo en el que fue capturado el iésimo cuadro.

Para determinar la fase instantánea generalmente se utilizan algoritmos que operan espacialmente, los cuales pueden llegar a ser muy exigentes computacionalmente, muy sensibles al ruido y condiciones de frontera, y necesitan la adición de dispositivos moduladores de fase (Chen, 2008). En cambio, es posible asumir que la evolución temporal de la señal de cada píxel es totalmente independiente de los demás, y de esta forma, el análisis se puede pasar del dominio espacial bidimensional al dominio temporal unidimensional (Madjarova et al., 2002).

Además, se puede asumir que la señal temporal de un píxel cualquiera durante el proceso de deformación se puede aproximar a una función analítica. Esta función, es de la forma $f(\phi(t))=$ $\cos (\phi(t))$ a la cual se le asocia su función conjugada a través de las propiedades de la transformada de Hilbert. De esta manera, se tiene que $\mathcal{H}\{f(\phi(t))\}=\sin (\phi(t))$ y se puede obtener la fase por medio de la siguiente ecuación (Madjarova et al., 2003; Toyooka et al., 2006):

$\phi(t)=\tan ^{-1}\left(\frac{\mathcal{H}\{f(\phi(t))\}}{f(\phi(t))}\right)$.

Es importante notar que para poder calcular la fase a partir de (2) se requiere eliminar la intensidad $I_{0}\left(x, y, t_{i}\right)$ en (1). Este proceso se realiza restando a cada píxel el valor promedio de los píxeles vecinos dentro de una ventana espacio-temporal con el tamaño suficiente para eliminar esta intensidad de fondo sin introducir ruido (Madjarova et al., 2002).

La fase $\phi(t)$ obtenida en (2), por propiedades de la tangente inversa, solo tiene valores entre $-\pi$ y $\pi$, lo que se conoce como fase envuelta. Es necesario realizar procedimientos de desenvolvimiento para tener valores continuos. Sin embargo, gracias a que la 
señal temporal es unidimensional, el desenvolvimiento se realiza fácilmente con algoritmos comunes como los que se encuentran en MATLAB.

Para obtener la fase producida por la deformación, basta con restar cada mapa instantáneo de fase con la fase del cuadro referencia. De esta forma también se elimina la fase de speckle, que varía mucho más lento que la fase de la señal, y se puede tomar como constante durante periodos de tiempo significativos. Se tiene entonces:

$\Delta \phi\left(x, y, t_{i}\right)=\varphi\left(x, y, t_{i}\right)-\varphi\left(x, y, t_{R}\right)$

Donde $\varphi\left(x, y, t_{R}\right)$ corresponde a la fase referencia, la cual debe ser refrescada o actualizada periódicamente para evitar los efectos que tiene la variación temporal de la fase de speckle.

La fase final obtenida es proporcional a la componente de deformación en la dirección en la cual se realizó el desplazamiento de los dos patrones de speckle en el interferómetro. Esta proporcionalidad permite tener una medida cualitativa de las deformaciones y el esfuerzo instantáneo, los cuales son de gran utilidad práctica. Los factores de proporcionalidad se pueden establecer específicamente para cada ensayo, pero no son necesarios en la mayoría de las aplicaciones. El ruido remanente en los mapas de fase es tratado utilizando filtros de mediana.

\section{METODOLOGía}

El objeto de estudio fue una lámina circular de acero, con un espesor de $1 \mathrm{~mm}$, la cual estaba fija en sus bordes a un marco igualmente circular. La deformación de la lámina se realizó a través de carga térmica con una lámpara halógena que se puso en la parte posterior de la lámina. Se utilizó LabVIEW para hacer un programa de adquisición de imágenes. El programa iniciaba al momento de encenderse la lámpara halógena y almacenaba la secuencia de imágenes de todo el proceso de calentamiento. Cada secuencia tomada tuvo una duración de 21 segundos, a una tasa de 10 cuadros por segundo. 
Las secuencias de imágenes se procesaron digitalmente en MATLAB, en donde eran convertidas en una matriz de datos 3D. Luego se procesó la señal de cada píxel independiente mente para obtener la fase instantánea en cada punto. Se utilizaron los comandos propios del software para realizar la transformada de Hilbert, la tangente inversa, el desenvolvimiento de la fase y los filtros espaciales para la eliminación del ruido remanente. Finalmente, se reconstruyó la secuencia con la evolución del mapa de fase durante todo el proceso de deformación.

\section{RESULTADOS Y DISCUSIÓN}

En la Fig. 2 se muestran tres cuadros en tres instantes diferentes del proceso de calentamiento. En primer lugar se observan las franjas y su evolución, y luego, la fase en niveles de gris, donde negro es 0 y blanco es $2 \Pi$. Los mapas de fase obtenidos muestran en las zonas brillantes los puntos en donde la lámina sufrió una mayor deformación, mientras las zonas oscuras fue donde no hubo deformación, respecto a la dirección de derivación.

Es importante notar que dada la naturaleza par de la función coseno y los algoritmos internos de MATLAB para calcular la función tangente inversa, no fue posible recuperar el signo de la fase. De esta forma, los mapas de fase corresponden a los valores absolutos de la misma. Sin embargo, la información obtenida sigue siendo muy importante puesto que permite detectar las zonas de mayor deformación y la presencia de fallas y defectos, durante un proceso dinámico. Esto permite el control de calidad en muchas industrias, así también como la inspección de elementos de deformación programada.

Es importante destacar que el análisis temporal reduce notoriamente el volumen y la complejidad de los algoritmos de procesamiento. En particular, el desenvolvimiento de fase unidimensional se pudo realizar fácilmente y sin necesidad de tener en cuenta el ruido ni las discontinuidades. Además, el sistema se implementó sin la necesidad de sistemas antivibratorios ni protección contra contaminación lumínica. De esta forma, puede ser adaptado a condiciones industriales fácilmente. 


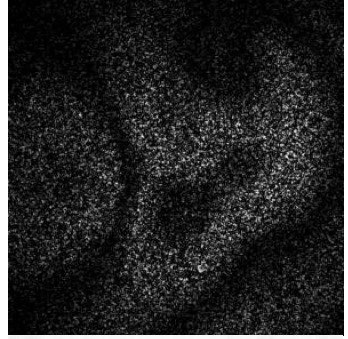

$t=2 \mathrm{~s}$

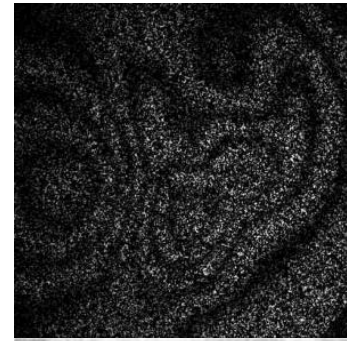

$t=10 \mathrm{~s}$

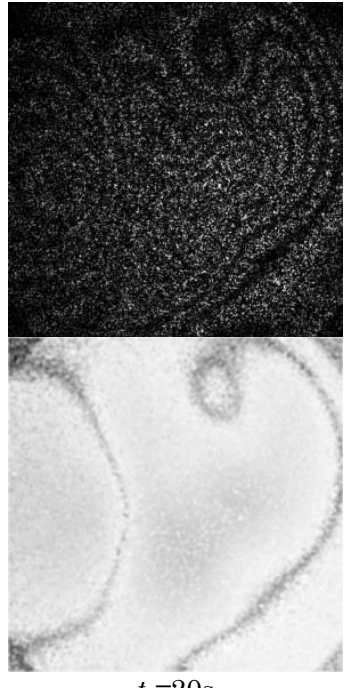

$t=20 \mathrm{~s}$

Fig. 2. Evolución temporal del diagrama de franjas, secuencia superior, y del mapa de fase, secuencia inferior, del proceso de deformación térmica. La fase está en valores de la escala de gris, donde el negro corresponde a $0 \mathrm{rad}$ y el blanco a 2 п $\mathrm{rad}$

\section{CONCLUSIONES}

Se implementó un sistema de shearografía para el análisis de deformaciones durante procesos dinámicos de tensión térmica. El sistema utiliza herramientas para el análisis temporal que permiten reducir y simplificar la cantidad de cálculos requeridos para procesar las imágenes.

Se obtiene la evolución instantánea del mapa de fase durante todo el proceso. Los valores en estos mapas, sin embargo, corresponden al valor absoluto de la fase, dado que los algoritmos utilizados no son capaces de discriminar el cuadrante del ángulo. Es necesario entonces, implementar estrategias y algoritmos alternativos para evitar la ambigüedad de la paridad de la función coseno.

Sin embargo, la herramienta desarrollada permite discriminar las regiones dentro del área de interés según la deformación sufrida. Además, permite la inspección de deformaciones programadas 
y fallas y defectos del material que sean notorios solo a bajo ciertos rangos de estrés.

\section{AGRADECIMIENTOS}

Este trabajo se pudo desarrollar gracias al apoyo del Instituto Tecnológico Metropolitano, a través del proyecto P13142, y de la Universidad Nacional de Colombia - Sede Medellín.

\section{REFERENCIAS}

Chen, J. (2008). Improved phase-shifted digital speckle shearography for time-dependent deformation measurement. Optical Engineering, 47(6), 065601.

Hung, Y. Y. (1996). Shearography for non-destructive evaluation of composite structures. Optics and Lasers in Engineering, 24(2-3), 161-182.

Hung, Y. Y., \& Ho, H. P. (2005). Shearography: An optical measurement technique and applications. Materials Science and Engineering: R: Reports, 49(3), 61-87.

Kalms, M. (2006). Mobile shearography for ndt of technical and artwork components. ndt.net. Retrieved from http://www.ndt.net/article/apcndt2006/papers/20.pdf

Krupka, R., Walz, T., \& Ettemeyer, A. (2005). Industrial applications of shearography for inspection of aircraft components. In J. F. Lopez, C. Quan, F. S. Chau, F. V. Fernandez, J. M. Lopez-Villegas, A. Asundi, ... C. T. Lim (Eds.), Microtechnologies for the New Millennium 2005 (pp. 476-479). International Society for Optics and Photonics.

Lobanov, L. M., Pivtorak, V. a., Kyyanets', I. V., \& Savyts'ka, O. M. (2007). Nondestructive testing of composite and metallic pipes by the method of electronic shearography. Materials Science, 43(4), 522-527.

Madjarova, V., Kadono, H., \& Toyooka, S. (2003). Dynamic electronic speckle pattern interferometry (DESPI) phase analyses with temporal Hilbert transform. Optics express, 11(6), 617-23.

Madjarova, V., Toyooka, S., Widiastuti, R., \& Kadono, H. (2002). Dynamic ESPI with subtraction-addition method for obtaining the phase. Optics communications, 212(October), 35-43. Retrieved from http://www.sciencedirect.com/science/article/pii/S0030401802019090

Neuman, J. W. (2008). Aerospace NDT with advanced laser shearography. 17th World Conference on Nondestructive Testing, 1-6. Retrieved 
from http://femtosecondsystems.com/Aerospace NDT with Advanced Laser Shearogra-phy/Aerospace NDT with Advanced Laser Shearography_534.pdf

Osten, W. (2003). Mobile shearography system for the inspection of aircraft and automotive components. Optical Engineering, 42(5), 1188. doi:10.1117/1.1566968

Scalea, F. L. Di, Spicer, J. B., \& Green, R. E. (2000). Electronic Shearography with Thermal Loading for Detecting Debonds in Thick Polyurethane/Steel Panels for Marine Applications. Research in Nondestructive Evaluation, 12(1), 43-51.

Steinchen, W., \& Yang, L. (2003). Digital Shearography: Theory and Application of Digital Speckle Pattern Shearing Interferometry ( $p$. 330). SPIE Publications.

Toyooka, S., Kadono, H., Saitou, T., Sun, P., Shiraishi, T., \& Tominaga, M. (2006). Dynamic ESPI system for spatio-temporal strain analysis. In W. Osten (Ed.), Fringe 2005 (pp. 656-661). Springer Berlin Heidelberg. 